\title{
El gusano blanco de la papa. Premnotrypes vorax (Hustache) en Colombia: II.- fluctuación de poblaciones de larvas en el campo
}

\section{Luis Valencia V.*}

\begin{abstract}
RESUMEN
Los resultados que se presentan fueron obtenidos de experimentos de campo realizados durante 1988 en el lote 5 del Centro Nacional de Investigación "Tibaitatá" del Instituto Colombiano Agropecuario (ICA), cerca de Bogotá. Se investigó la fluctuación de las poblaciones de larvas de gusano blanco en un campo sembrado con el cultivar Monserrate en el que se encontró que las larvas aparecen en el campo con el inicio de la tuberización y aumentan conforme aumenta el peso fresco de los tubérculos de la planta. En otro experimento plantado con 3 cultivares de diferente período vegetativo (precoz, semiprecoz y tardío), se encontró que hubo sobreposición de generaciones. Las evaluaciones se hicieron una vez por semana a partir de los 40 días de la siembra practicando una cosecha secuencial. Los resultados se discuten con base en literatura de reciente publicación.
\end{abstract}

Palabras Claves Adicionales: Gusano blanco, gorgojo de los Andes, Premnotrypes vorax, larvas, ecología, cosecha secuencial.

Aceptado para publicación: Julio 18, 1989.

* Entomólogo, Región I Zona Andina, Centro Internacional de la Papa, CIP.

Apartado Aéreo 92654 Bogotá, Colombia. 


\section{ABSTRACT}

\section{The Potato Andean Weevil, Premnotrypes vorax (Hustache) in Colombia. Il.- Fluctuation of Larvae Populations in the Field}

The results which are reported here, were obtained in 1988 from experimental plots (field No. 5) located at the Tibaitata farm of the Instituto Colombiano Agropecuario (ICA), near Bogotá. The population fluctuation of Andean Weevil larvae in a field sowed with the Monserrate cv, through the whole season was investigated. The larvae appeared infesting the potato tubers as early as the tuber seting begun. The increase in fresh weight for tubers (tuber seting curve) is closely followed for an increase in larvae number. In another experiment 3 different cultivars having different vegetative periods (early, semi early and late cultivars) were sown in the same plot. The overlapping of generations for the Andean Weevil was found in this plot. Evaluations were made once a week starting 40 days after the sowing date, with a sequential harvest. Results are discussed based on literature recently published.

Additional Index Words: Gusano blanco; white grub; Andean weevil; Premnotrypes vorax; larvae; ecology; sequential harvest.

No obstante es bien conocido el ciclo de vida del gusano blanco (GB) de la papa, Premnotrypes vorax (Hustache), (Zenner de Polanía y Posada, 1968; Calvache, 1986) bajo las condiciones de algunas localidades de Colombia, se conoce muy poco de la fluctuación de la población de larvas de esta especie a través de todo el ciclo del cultivo. Esta información es muy importante si se considera que las larvas del GB son realmente las que producen el daño en los tubérculos. En 1988 se condujeron experimentos con este objetivo y los resultados obtenidos se exponen y discuten en el presente artículo.

\section{MATERIALES Y MÉTODOS}

En el primer semestre (Semestre A) de 1988 se efectuaron 2 experimentos ( $\&$ \& B), en los cuales se investigó la infestación de los tubérculos por las larvas de GB. 


\section{Experimento A.-}

Las observaciones se realizaron en un lote de un cuarto de hectárea sembrado con papa del cultivar Monserrate. En este lote no se hizo ninguna aplicación de pesticidas y había sido sembrado previamente con 2 cultivos sucesivos de papa a fin de incrementar el nivel natural de infestación de GB. Las evaluaciones de la tuberización y de la infestación se iniciaron 40 días después de la siembra. Las evaluaciones se hicieron practicando cosechas secuenciales de 5 plantas distribuidas al azar una vez a la semana. En cada evaluación se registró el número y el peso de los tubérculos y el número de larvas de GB que se encontraban infestando los tubérculos así como aquéllos que se encontraban en el suelo circundante a la zona de tuberización.

\section{Experimento B.-}

En este experimento se investigó la infestación de las larvas de GB en 3 cultivares (cv.) diferentes de papa. En parcelas de 6 surcos por $10 \mathrm{~m}$ de largo se plantaron 2 surcos con un cultivar precoz (Criolla o Yema de huevo), 2 con un cv. semi-precoz (Monserrate) y 2 con un cv. tardío (Parda pastusa). Los períodos vegetativos de estos 3 cultivares fueron 110, 130 y 150 días, respectivamente. Se sembraron 10 parcelas con el arreglo ya mencionado y las evaluaciones fueron idénticas a las mencionadas en el experimento $A$, excepto que en este experimento las 5 plantas usadas para la evaluación de cada cultivar fueron contiguas y se tomaron al azar entre las 10 repeticiones.

\section{RESULTADOS}

Los resultados del experimento $A$ se muestran en la Figura 1. Se observa que el pico poblacional de las larvas de GB coincide con el pico de la curva de tuberización del cultivar Monserrate. En esta parcela experimental se registra sólo una generación de larvas. También se observa que las larvas de GB aparecen casi simultáneamente con el inicio de la tuberización.

Los resultados del experimento $B$ se muestran en la Figura 2. En estas parcelas se observan 2 picos poblacionales de las larvas de GB en los cultivares Criolla y Monserrate, excepto en el pico del día 113 para el cultivar Monserrate (que parece ser un error del muestreo), mientras que el cultivar Parda pastusa sólo mostró el segundo pico poblacional de las larvas. Por falta de lluvias en la última parte del ciclo del cultivo el cv. Parda pastusa se maduró rápidamente y estuvo lista para cosecha a los 145 días después de la siembra. 


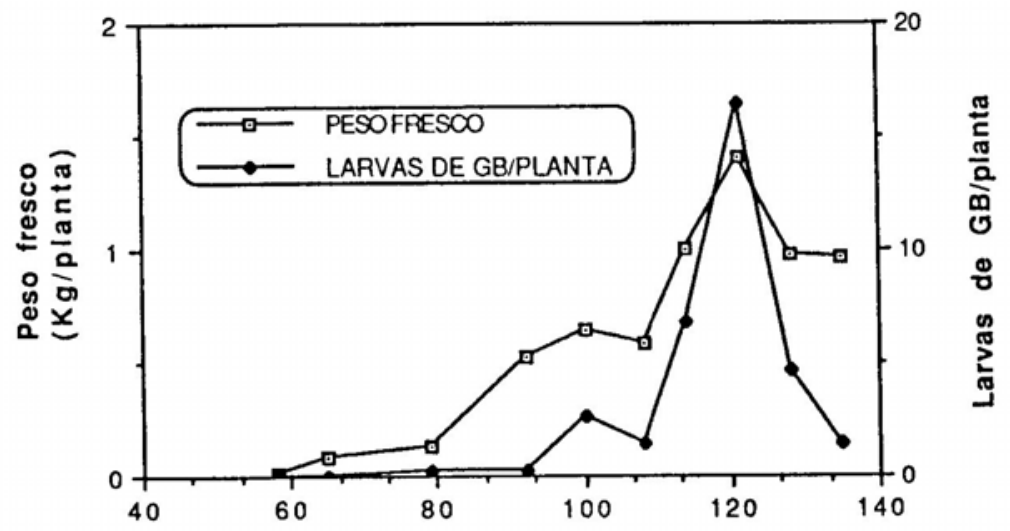

Edad del cultivo (dias)

FIGURA 1. Relación entre la tuberización del cultivar Monserrate y la infestación de las larvas de gusano blanco.

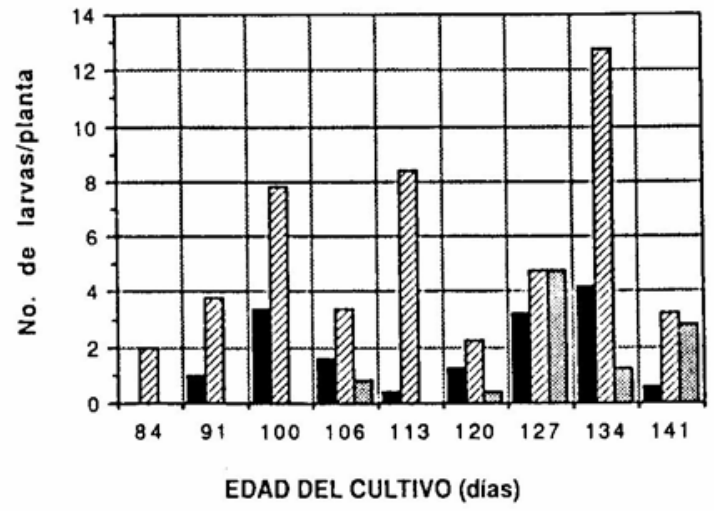

I CRIOLLA

ש MONSERPATE

-

FIGURA 2. Fluctuación de la población de larvas de gusano blanco en 3 cultivares de papa. 
Los resultados obtenidos en el experimento A sobre la investigación de la relación de la tuberización del cultivar Monserrate y la infestación por las larvas de GB, demuestran una clara sincronización del GB y la fenología del cultivo. Las larvas del GB aparecen justo cuando se inicia el proceso de tuberización y de allí su incremento sigue muy de cerca a la curva de tuberización para alcanzar su pico a los 120 días de la siembra con casi 17 larvas por planta.

Los resultados obtenidos en el experimento $B$, mediante el cual se investigó la curva de tuberización de 3 cultivares diferentes y la infestación por las larvas de GB, demuestran que hay sobreposición de generaciones en esta especie. Esta sobreposición de generaciones podría originarse por alguna de las 2 siguientes razones: Primero, se considera que el ritmo de oviposición de los adultos de GB es un proceso muy irregular con picos y bajadas muy pronunciadas, tal como lo demostró Alcázar (1976) para $P$. suturicallus Kuschel, en la Sierra Central del Perú. En este lugar los picos de producción de huevos pueden estar separados por 30 días o más, este lapso de tiempo fácilmente explicaría la presencia de 2 picos en la población de larvas.

La otra causa de la sobreposición de generaciones podría deberse a la existencia en el mismo campo de 3 cultivares con características muy diferentes. Al inicio del período vegetativo, los cultivares Criolla y Monserrate (precoz y semi-precoz, respectivamente) germinaron primero $y$ ejercieron una gran atracción a los adultos libres que se encontraban en los alrededores de sus respectivas parcelas, dejando muy pocos adultos para el cultivar Parda pastusa que germinó 12 días más tarde. La población de adultos que fue atraída a los cultivares Criolla y Monserrate dieron origen al primer pico de larvas y el segundo pico podría ser generado por la irregularidad en el ritmo de oviposición de las hembras o por los otros adultos que llegaron (migrantes o que emergieron del mismo campo en esos días) al cultivar Parda pastusa.

La presencia de la sobreposición de generaciones en miembros de la familia Curculionidae, ha sido reportada para Phyrdenus muriceus (Germ.) por Espul y Magistretti (1969) y para Graphonathus leucoloma Boheman por Todd (1964).

Por otro lado, se sabe que entre más largo es el ciclo de vida de un insecto, la sobreposición de generaciones se hará más pronunciada (Clark et al. 1974). 
De los resultados presentados aquí se deduce que el inicio de la tuberización es la etapa crítica para el control de las larvas del GB. Este inicio de la tuberización varía con el cultivar, calidad de semilla y la presencia de lluvias; por lo tanto, si se quiere controlar las larvas de GB con productos químicos, éste tendría que iniciarse en fechas cercanas al inicio de la tuberización.

En la actualidad se están conduciendo más experimentos en los cuales se trata de integrar los conocimientos básicos obtenidos en estos 3 años para tratar de mejorar el control del GB.

\section{Agradecimientos}

El autor agradece al personal de apoyo de la oficina regional del CIP en Bogotá (Región I) y al ICA por las facilidades prestadas para la realización de esta investigación.

\section{REFERENCIAS BIBLIOGRÁFICAS}

1. Alcázar, J. G. 1976. Biología y comportamiento del "Gorgojo de los Andes" Premnotrypes suturicallus Kuschel (Coleóptera: Curculionidae). Tesis de Ingeniero Agrónomo. Universidad Nacional del Centro del Perú. HuancayoPerú. 80 p.

2. Calvache, H. 1986. Aspectos biológicos y ecológicos del gusano blanco de la papa Premnotrypes vorax (Hustache). En: Memorias del Curso sobre Control Integrado de Plagas de Papa. L. Valencia ed. CIP-ICA, Colombia, pp. 18-24.

3. Clarck, L. R.; Geier, P. W.; Hughes, R. D.; Morris, R. F 1974 The ecology of insect populations in theory and practice. Methuen \& Co. and Science Paperbacks $232 \mathrm{p}$.

4. Espul, J. C.; Magistretti, G. 1969. Bioecología del "Gorgojo del tomate", Phyrdenus muriceus (Germ.) y su control en la provincia de Mendoza. Revista de Investigaciones Agropecuarias, INTA, Buenos Aires, Rep Argentina. Serie 5, Patología Vegetal, VI (5): 95-117.

5. Todd, D. H. 1964. Biology and control of white-fringed weevil. Proc. 17th., N. Z. Weed Pest. Control. Conf. 17: 125-129.

6. Zenner de Polanía, I.; Posada, L. 1968. Generalidades sobre el gusano blanco de la papa, Premnotrypes vorax (Hustache). Bogotá, Colombia. Agricultura Tropical 24(1): 33-40. 